\title{
Electric distribution network reconfiguration for power loss reduction based on runner root algorithm
}

\author{
Thuan Thanh Nguyen \\ Faculty of Electrical Engineering Technology, Industrial University of Ho Chi Minh City, Viet Nam
}

\begin{tabular}{l}
\hline \hline Article Info \\
\hline Article history: \\
Received Oct 27, 2019 \\
Revised Apr 8, 2020 \\
Accepted Apr 23, 2020 \\
\hline Keywords: \\
Distribution network \\
Network reconfiguration \\
Power loss \\
Radial topology \\
Runner root
\end{tabular}

\begin{abstract}
This paper proposes a method for solving the distribution network reconfiguration (NR) problem based on runner root algorithm (RRA) for reducing active power loss. The RRA is a recent developed metaheuristic algorithm inspired from runners and roots of plants to search water and minerals. RRA is equipped with four tools for searching the optimal solution. In which, the random jumps and the restart of population are used for exploring and the elite selection and random jumps around the current best solution are used for exploiting. The effectiveness of the RRA is evaluated on the 16 and 69-node system. The obtained results are compared with particle swarm optimization and other methods. The numerical results show that the RRA is the potential method for the NR problem.
\end{abstract}

Copyright $\odot 2020$ Institute of Advanced Engineering and Science. All rights reserved.

\section{Corresponding Author:}

Thuan Thanh Nguyen,

Faculty of Electrical Engineering Technology,

Industrial University of Ho Chi Minh City,

No. 12 Nguyen Van Bao, Ward 4, Go Vap District, Ho Chi Minh City, Viet Nam.

Email: nguyenthanhthuan@iuh.edu.vn

\section{INTRODUCTION}

Electric distribution system (EDS) has mesh topology but it is usually operated in radial topology due some advantages such as reduction of short-circuit current and installation of protect devices. However, radial operation takes more power loss compared with mesh operation. Therefore, reduction of power loss in EDS takes a high role in operating EDS. In among methods of reduction power loss, network reconfiguration (NR) is the most efficient technique because of no costs. It is performed by changing the status of opened and closed switches in EDS.

The NR problem is first proposed in [1]. In this study, the NR problem is formulated by a mixed integer non-linear problem and solved by a discrete branch-and-bound technique. Then, Civanlar et al., [2] solved the NR problem based on exchanging switches to reduce power loss. After almost four decades, the NR problem has solved by many modern methods stimulated from phenomena of nature or society such as genetic algorithm (GA), particle swarm optimization (PSO), fireworks algorithm (FWA) and cuckoo search algorithm (CSA), biogeography based optimization (BBO), grey wolf optimization (GWO). In [3-5], GA has been used to solve the NR problem for minimizing power loss. In [6-8], PSO is proposed for solving the NR problem to reduce power loss. In $[9,10] \mathrm{FWA}$ is proposed for the NR problem to reduce power loss and improve the node voltage. In [11-13], CSA has been successful solved the NR problem for reducing power loss and improving node voltage. In [14] modified BBO is successful applied for finding the optimal configuration for power loss reduction. The GWO is also successful applied for the NR problem to reduce power loss $[15,16]$. In comparison with the heuristic methods which are based on the knowledge of electric power system such as [1, 2], the modern methods have more advantages. While the heuristic methods are only usually to optimize the radial topology for power loss reduction, the modern methods called metaheuristic methods are easy used to optimize the radial topology for different type of objective such as 
power loss reduction, voltage improving, reliability improving or multi-objective. Furthermore, the obtained result of the heuristic methods is usually local extremes but the meta-heuristic methods have ability to provide good solution for the NR problem. In recent years, many algorithms belonging to this method group are being developed. Therefore, the study of applying new algorithms to the NR problem is also a matter of great concern to find out and contribute more effective methods for the NR problem.

Runner root algorithm (RRA) is a recent meta-heuristic method inspired from runners and roots of plants to search water and minerals [17]. To explore the search space, RRA uses random jumps technique with high steps to generate the new solutions far from current solutions and the re-initialization technique to restart the current population. To exploit the search space, RRA uses random jumps technique with small steps to generate new solutions around the current best solution and the elite selection technique to save the current best solution for next generation. For solving twenty-five benchmark functions, RRA has demonstrated advantages compared to others methods [17]. For application of RRA for the problems of the power system, RRA have been successful applied for the NR problem with multi-objective function [18] and the placement of DG in the EDS [19]. In this paper, RRA is adapted to solve the NR problem for power loss reduction. The performance of RRA is tested in different EDS and compared with the well-known PSO that has been successful applied for the NR problem $[8,20]$. In addition, the calculated results obtained by RRA are also compared to other methods in literature. The highlights of the paper is summarized as follows:

- $\quad$ RRA is adapted for solve the NR problem for power loss reduction;

- $\quad$ RRA outperforms PSO and other methods in literature in terms of successful rate and the quality of the obtained optimal solution.

In the bellowing section, the problem formulation is presented. The application of RRA for the NR problem is presented in section 3. Section 4 shows the results and analysis and finally conclusions are listed in section 5 .

\section{PROBLEM FORMULATION}

The purpose of the NR is transferring a part of loads from the heavy branches to light branches by changing the opened/closed status of switches located on each branch. Power loss of EDS is calculated by sum of power loss of each branch of the system. However, there are closed branches carrying current and opened branches not carrying current in the EDS. Therefore power loss of the EDS is calculated by as follows:

$$
\Delta P=\sum_{i=1}^{N b r} k_{i} R_{i} \frac{P_{i}^{2}+Q_{i}^{2}}{V_{i}^{2}}
$$

In which, $\mathrm{Nbr}$ is a number of branches of EDS, $R_{i}$ is the ith branch's resistance. $\mathrm{P}_{\mathrm{i}}$ and $\mathrm{Q}_{\mathrm{i}}$ are the active and reactive power flow on the ith branch. $k_{i}$ stands for the status of the branch ith in the EDS which is equal to 1 for closed status and 0 for vice versa.

The results of NR problem is a radial topology of EDS that satisfy following constraints:

- $\quad$ Radial topology constraint: To satisfy this constraint, the empirical formula [21] is proposed to check candidate solutions.

$$
\operatorname{det}(A)= \begin{cases}-1 \text { or } 1, & \text { radial } \\ 0, & \text { not radial }\end{cases}
$$

In which, $\operatorname{det}(\mathrm{A})$ is determinant of matrix $\mathrm{A}$. A is the branch by node matrix built by connection of EDS.

- $\quad$ Node voltage constraint: node voltage magnitude must lie in permissible ranges $\left[V_{\min }, V_{\max }\right]$. They are respectively set equal to $[0.95,1]$ in per unit.

$$
V_{\min } \leq V_{j} \leq V_{\max } \text { with } j=1,2, \ldots \text { Nbus }
$$

where $N$ bus is the number of nodes in the EDS.

- Branch current constraint: For avoiding over load, the branches' current magnitude must lie in their permissible range.

$$
I_{i} \leq I_{\max , i} \text { with } i=1,2, \ldots \mathrm{Nbr}
$$




\section{RRA FOR THE NR PROBLEM WITH POWER LOSS REDUCTION}

RRA is inspired from the plants propagated through runners and roots. To apply this as an optimization tool, Merrikh-Bayat used three idealized rules [17]:

- $\quad$ The mother plant is generated the daughter plant through its runner for exploring resources.

- The plants produce roots and root hairs to exploit resources around its position.

- The daughter plants will grow faster and become the mother plant at new position with rich resources.

Otherwise, they will be die at new position with poor resources.

In this study, the implementation of RRA for the NR problem is summarized as follows:

- $\quad$ Step 1: Initialization

For solving the NR problem, each mother plant is considered as radial topology of the distribution system. In the first step, the population of the problem is generated as (5). In which, each radial topology is presented as (6) and each variable of candidate solution which is an open switch is generated randomly as (7).

$$
\begin{aligned}
& \text { population }=\left\{\begin{array}{l}
X_{\text {mother }, 1} \\
X_{\text {mother }, 2} \\
\ldots \\
X_{\text {mother }, N}
\end{array}\right. \\
& X_{\text {mother }}(k)=\left[X_{1}, X_{2}, \ldots, X_{d}, \ldots, X_{\text {dim }}\right] \\
& X_{d}(k)=\operatorname{round}\left[X_{\text {low }, d}+\text { rand } \times\left(X_{\text {high }, d}-X_{\text {low }, d}\right)\right]
\end{aligned}
$$

where, $\mathrm{k}=1,2, \ldots, \mathrm{N}$ and $\mathrm{d}=1,2, \ldots, \operatorname{dim}$ with $\mathrm{N}$ and $\operatorname{dim}$ are respectively population size and the number of variables. $X_{\text {low,d }}$ and $X_{\text {high,d }}$ are respectively low and high limit of tie-switch Xd. Based on the initialized population of the mother plants, each mother plan is evaluated by the fitness function and the plant with the best fitness function is saved to the best daughter plant $X_{\text {daughter,best }}$. Noted that, to calculate the fitness function value, the power flow is performed and the value of the (1) is obtained.

- $\quad$ Step 2: Global search with generation of daughter plants

To explore search space, a new population of daughter plants is generated to replace the population of mother plants.

$$
X_{\text {daughter }}(k)=\left\{\begin{array}{l}
X_{\text {daughter, } \text { best }, k}, k=1 \\
\text { round }\left[X_{\text {mother }, k}(k)+d_{\text {runner }} \times \text { rand }\right], k=2, \ldots, N
\end{array}\right.
$$

where, the constant parameter $d_{\text {runner }}$ is a large distance between the mother and daughter plant. Then, the fitness function of each daughter plant is evaluated and a best daughter plant $\left(X_{\text {daughter,best }}\right)$ is updated.

- $\quad$ Step 3: Local search with large and small distances

To exploit search space, this step is performed as the value of the best daughter plant in two generations is not improved considerably. The best daughter plant will generate dim new plants by modifying one by one element in the best daughter plant. The first dim new plants are generated around the best daughter plant with large distances as follows:

$$
X_{\text {perturbed,d }}=\operatorname{round}\left[\operatorname{vec}\left\{1,1 \ldots, 1,1,1+d_{\text {runner }} \times \operatorname{rand}_{d}, 1, \ldots, 1\right\} \times X_{\text {daughter,best }}(i)\right]
$$

where vec $\left\{1,1 \ldots, 1,1,1+d_{\text {runner }} \times\right.$ rand $\left., 1, \ldots, 1\right\}$ is a vector with all elements are equal to 1 except for the $\mathrm{d}$-th one, which is equal to $1+d_{\text {runner }} \times$ rand $_{d}$. The second dim new plants are generated by replacing $d_{\text {runner }}$ with $d_{\text {root }}$, which is much smaller than $d_{\text {runner }}$. The new daughter plants are evaluated the fitness function and the best daughter plant is updated again.

- Step 4: Generation of new mother plants and escaping the local optimal

At the final stage of each iteration, based on the fitness of the daughter plants, the roulette wheel selection method [22] is used to selection the daughter plants as the mother plants for the next generation. Noted that the best daughter plant will has large probability selected for the next generation. In addition, to escape local optimal solution, a re-initialization strategy is used to restart the algorithm. If after Stall $\max$ generations that the value of the best daughter plant still no considerable improvement, the population of mother plants will be randomly generated similar to step 1 . The pseudo code of the RRA for the NR to minimize power is given in Figure 1. 


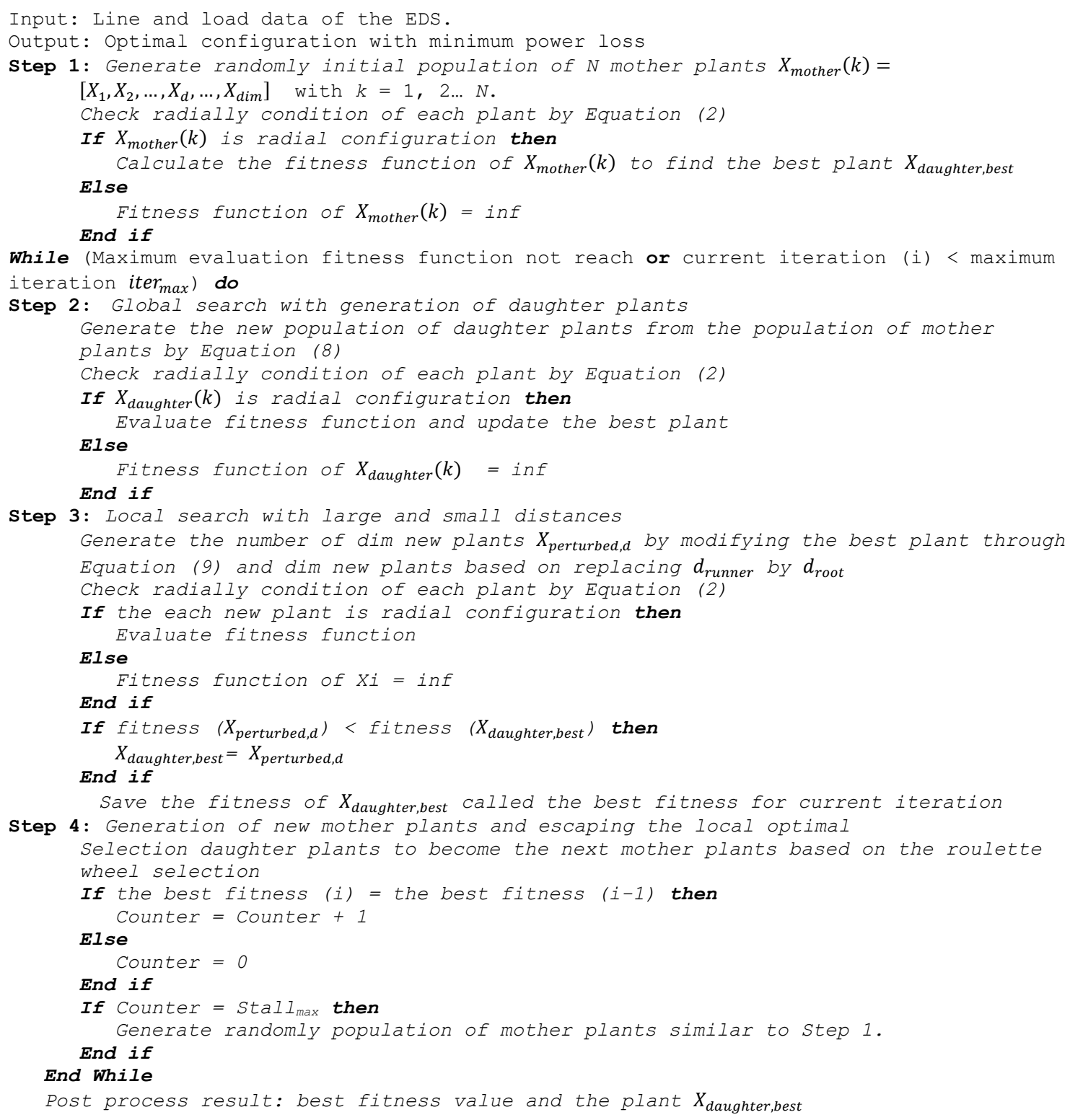

Figure 1. Pseudo code of the RRA for the NR problem for power loss reduction

\section{RESULTS AND ANALYSIS}

To show the efficient of RRA, the application of RRA for the NR problem is implemented in platform Matlab 2016a, executed on a personal computer Intel(R) Core(TM) i5-2430 CPU@2.4GHz with 4GB RAM. The performance of RRA is evaluated in two EDS consisting of 14-node and 69-node system. In addition, the application of PSO for the NR problem is also implemented and run on the same computer for comparing with RRA besides comparing RRA with other methods in literature.

\subsection{The 16-node system}

The $23 \mathrm{kV}$ 16-node test system shown in Figure 2 contains 3 feeders and 13 load nodes. The data of the system is referenced from [3]. The three initially-open switches are $\{$ S14, S15 and S16 $\}$. The total load of the system is $28.7 \mathrm{MW}$, while the initial total power loss is $511.4356 \mathrm{~kW}$. The minimal voltage amplitude $\left(\mathrm{V}_{\min }\right)$ of the system is $0.9693 \mathrm{p}$.u. The calculated time of the algorithm usually depends on the mechanism of operation of the algorithm. While some algorithms create new solutions using simple procedures, others produce new solutions using more complex and time-consuming procedures. But overall, increasing the population size $(\mathrm{N})$ the maximum number of iterations ( iter $_{\max }$ ) and the maximum number of fitness 
evaluation (MFE) to high values can help the algorithms to produce the higher number of new solutions in the searching space but it will take a long time for searching optimal solutions. Therefore, in this study based on the scale and complexity of the test system, the control parameters of RRA and PSO consisting of N, iter $_{\max }$ and MFE are experimented several times and chosen to 10, 50 and 500 respectively. For RRA, the $d_{\text {runner }}$ and $d_{\text {root }}$ are set to 4 and 2 respectively [18]. For PSO, two constants $C_{1}$ and $C_{2}$ in the velocity equation of particles to control the position of particle learn from its own and the best so far particle experience are set to $2[8,20]$.

The obtained results on the 16-node test system is presented in Table 1 . The power loss of the 16-node system has been decreased from $511.4356 \mathrm{~kW}$ to $466.1267 \mathrm{~kW}$ corresponding to $8.9 \%$ by using the RRA method. This power loss value is reached by opening the switches $\{$ S6, S12 and S14 $\}$ replacing for the switches $\{\mathrm{S} 14, \mathrm{~S} 15$ and S16\}. The minimal voltage amplitude has been increased from 0.9693 p.u. at the node 10 before reconfiguration to $0.97158 \mathrm{p}$.u. after performing reconfiguration. This results is identical to the results obtained by the genetic algorithm [3], modified tabu search algorithm (MTS) [21] and binary particle swarm gravity search algorithm (BPSOGSA) [23]. Noted that, although BPSOGSA has found out the optimal configuration but the average value of the fitness function (Fit ${ }_{\text {averge }}$ ) and the standard deviation (STD) of the fitness function are 479.2 and 28.9 which are 9.5083 and 21.0377 higher compared with those of RRA. The voltage of nodes in the 16-node test system after reconfiguration shown in Figure 3 demonstrates that most of voltage of nodes has been improved after opening the switches \{S6, S12 and S14 and no node violates the voltage constraints.

Similar to RRA, PSO has also determined the optimal configuration $\{\mathrm{s} 6, \mathrm{~s} 12$ and $\mathrm{s} 14\}$ but in 50 runs PSO has only achieved the optimal solution in 12 runs corresponding to $24 \%$ while RRA has reached the optimal configuration in 41 runs corresponding to $82 \%$ which is $58 \%$ higher than PSO. In 50 runs, the maximal (Fit ${ }_{\max }$ ), Fit ${ }_{\text {averge }}$ and STD of the fitness function values of RRA are lower than those of PSO. Their values of RRA are respectively 493.1542, 469.6917 and 7.8623 for RRA while for PSO they are 511.4356, 495.4369 and 18.6443 respectively. In addition, the executed times for RRA solving the16-node system reconfiguration is also faster than that of PSO. The mean, minimum and maximum convergence curves of proposed RRA and PSO in 50 runs are presented in Figure 4. As presented from the figure, RRA has better performance compared with PSO in terms of the optimal convergence value for 50 runs. These results demonstrate that RRA outperforms to PSO for the NR problem.

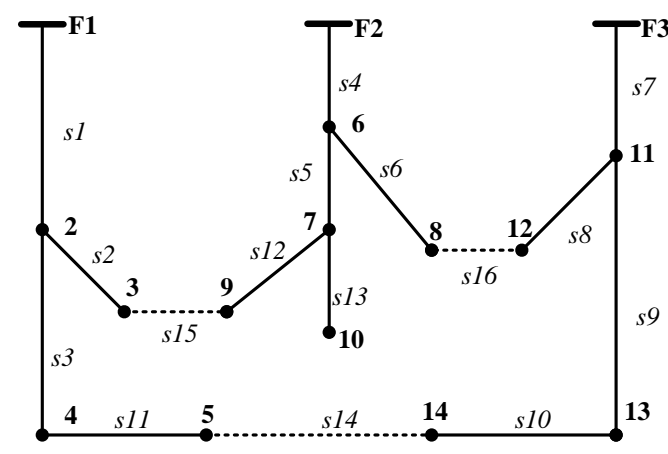

Figure 2. 16-node test system

Table 1. The comparisons among RRA with PSO and other methods for the 16-node system

\begin{tabular}{|c|c|c|c|c|c|c|}
\hline Item & Initial & RRA & PSO & GA [3] & MTS [21] & BPSOGSA [23] \\
\hline Optimal opened switches & $14,15,16$ & $6,12,14$ & $6,12,14$ & $6,12,14$ & $6,12,14$ & $6,12,14$ \\
\hline$\Delta \mathrm{P}(\mathrm{kW})$ & 511.4356 & 466.1267 & 466.1267 & 466.1267 & 466.1267 & 466.1267 \\
\hline $\mathrm{V}_{\min }$ (p.u.) (bus) & $0.9693(10)$ & $0.97158(10)$ & $0.97158(10)$ & $0.97158(10)$ & $0.97158(10)$ & $0.97158(10)$ \\
\hline $\mathrm{Fit}_{\min }$ & - & 466.1267 & 466.1267 & - & - & - \\
\hline Fit $_{\text {average }}$ & - & 469.6917 & 495.4369 & - & - & 479.2 \\
\hline STD of fit. & - & 7.8623 & 18.6443 & - & - & 28.9 \\
\hline $\begin{array}{l}\text { Average convergence } \\
\text { iterations }\end{array}$ & - & 20 & 3 & - & - & - \\
\hline Run time (sec) & - & 1.4891 & 1.7559 & - & - & - \\
\hline
\end{tabular}




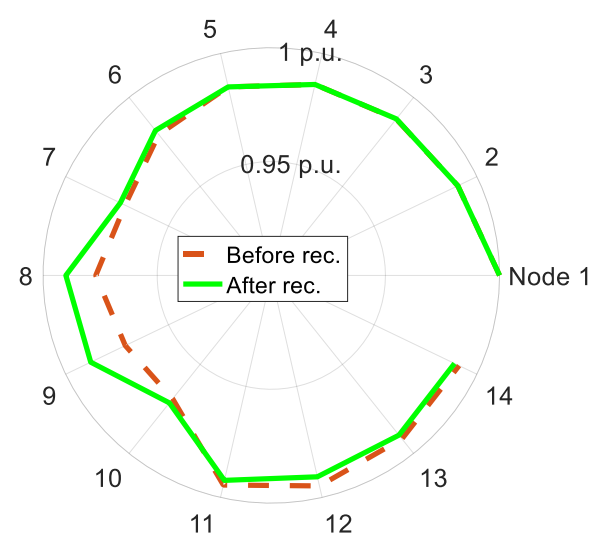

Figure 3. The voltage of nodes in the 16node test system after reconfiguration

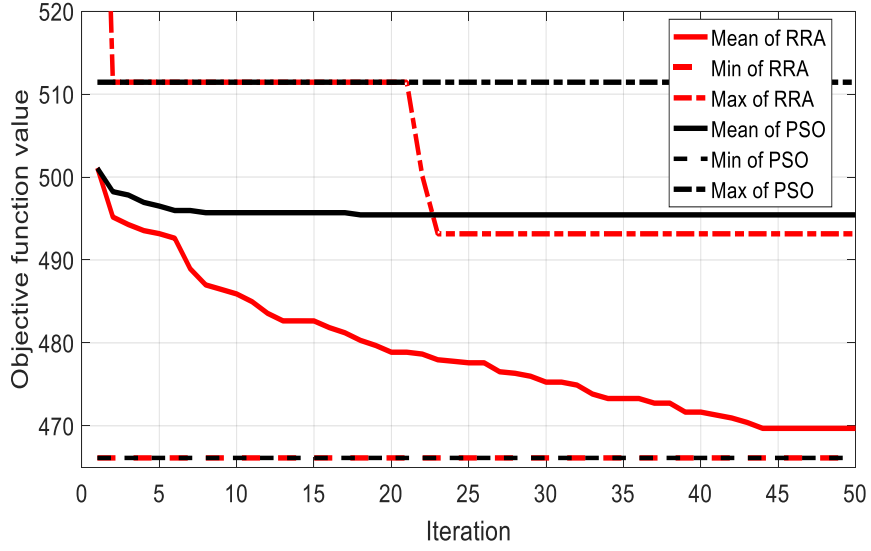

Figure 4. The convergence curves of RRA and PSO for 16-node test system

\subsection{The 69-node system}

The 12.66kV 16-node system shown in Figure 5 contains 1 feeders and 68 load nodes. The data of the system is referenced from [24]. The five initially-open switches are $\{$ S69, S70, S71, S72 and S73 $\}$. The total load of the system is $3.8015 \mathrm{MW}$, while the initial total power loss is $224.8871 \mathrm{~kW}$. The minimal voltage amplitude $\left(\mathrm{V}_{\mathrm{min}}\right)$ of the system is 0.9092 p.u. at the node 65 . The control parameters for both of RRA and PSO consisting of $\mathrm{N}$, iter max $_{\max }$ and MFE are set to 20, 150 and 3000 respectively. The values of the $\mathrm{d}_{\text {runner, }}$, $\mathrm{d}_{\text {root }}$ for RRA and $\mathrm{C}_{1}, \mathrm{C}_{2}$ for PSO are set similar to those in the 16-node system.

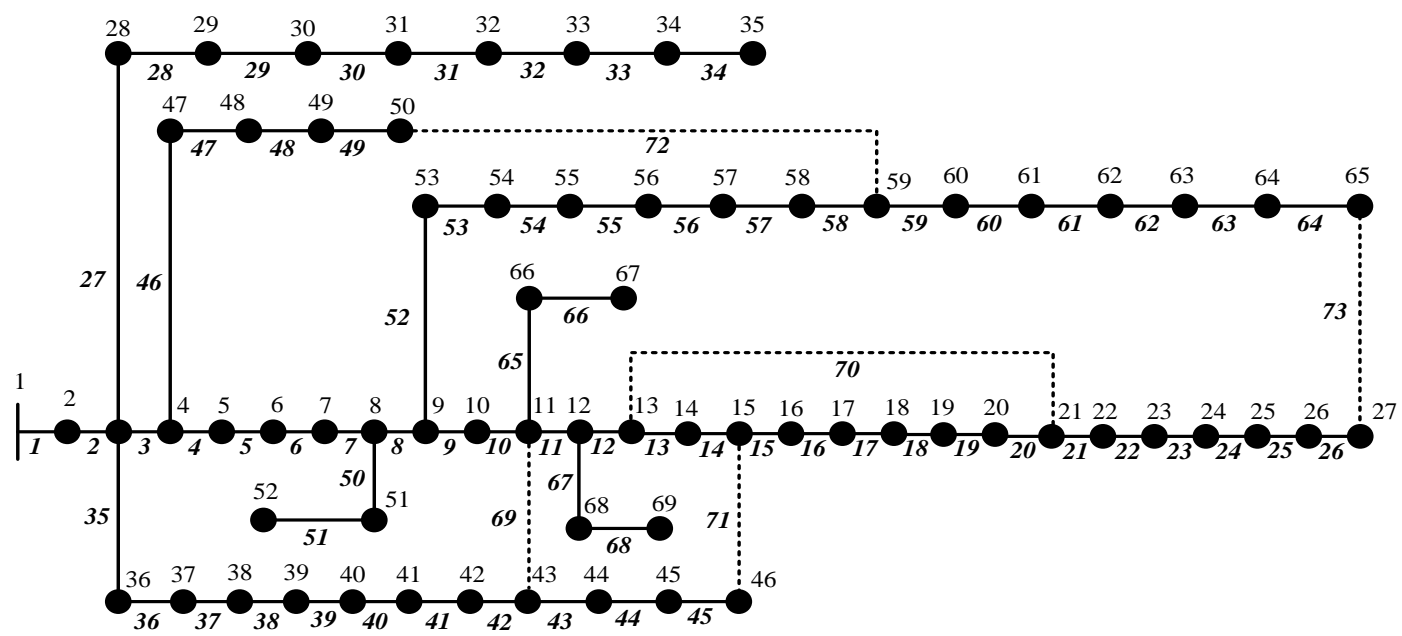

Figure 5. The 69-node test system

The obtained results on the 69-node test system is presented in Table 2. The power loss of the 69 -node system has been decreased from $224.8871 \mathrm{~kW}$ to $98.5875 \mathrm{~kW}$ corresponding to $56.16 \%$ by using the RRA method. This power loss value is reached by opening the switches $\{$ S69, S70, S14, S57 and S61\} replacing for the switches $\{$ S69, S70, S71, S72 and S73 $\}$. The minimal voltage amplitude has been increased from 0.9092 p.u. at the node 65 before reconfiguration to 0.9495 p.u. at the node 61 after performing reconfiguration. This results is identical to the results obtained by the cuckoo search (CSA) [13], adaptive shuffled frogs leaping algorithm (ASFLA) [25] and BPSOGSA [23] and better than harmony search

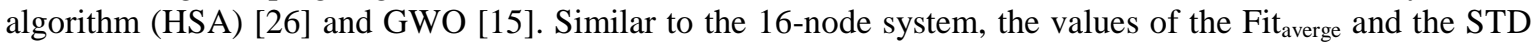
of BPSOGSA are 171.5 and 168.1 which are much higher compared with those of RRA. The voltage of nodes in the 69-node test system after reconfiguration shown in Figure 6 shows that most of voltage of nodes has been improved after opening the switches $\{$ S69, S70, S71, S72 and S73 \}. 
Table 2. The comparisons among RRA with PSO and other methods for the 69-node system

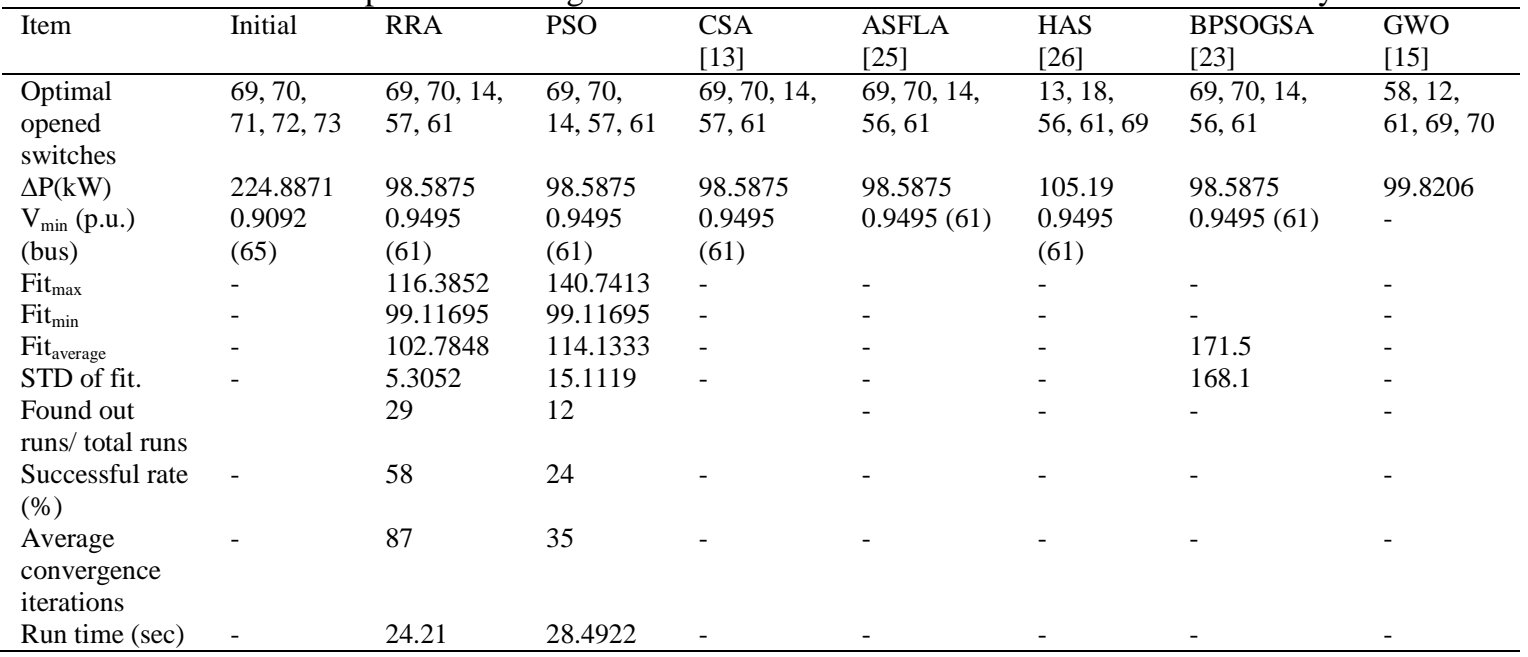

In comparison between RRA with PSO, for the 69-node system PSO has also obtained the optimal configuration $\{$ S69, S70, S14, S57 and S61 $\}$ but in 50 runs PSO has only returned the optimal solution in 12 runs corresponding to $24 \%$ while RRA has returned the optimal configuration in 29 runs corresponding to

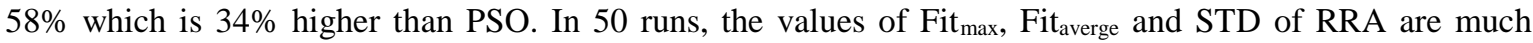
lower than those of PSO. Their values of RRA are respectively $116.3852,102.7848$ and 5.3052 for RRA while for PSO they are 140.7413, 114.1333 and 15.1119 respectively. In term of the number of average convergence iterations, although PSO has converged early than RRA but PSO converges usually to local solution. In addition, the executed times for PSO solving the 69-node system reconfiguration is also slower than that of RRA. The mean, minimum and maximum convergence curves of RRA and PSO in 50 runs are presented in Figure 7. As presented from the figure, RRA has better performance compared with PSO in terms of the optimal convergence value for 50 runs and the mean convergence curve of RRA is very closed to the minimum convergence curve and much lower than that or PSO. These results once again confirm that RRA is better than PSO and is the potential tool for the NR problem.

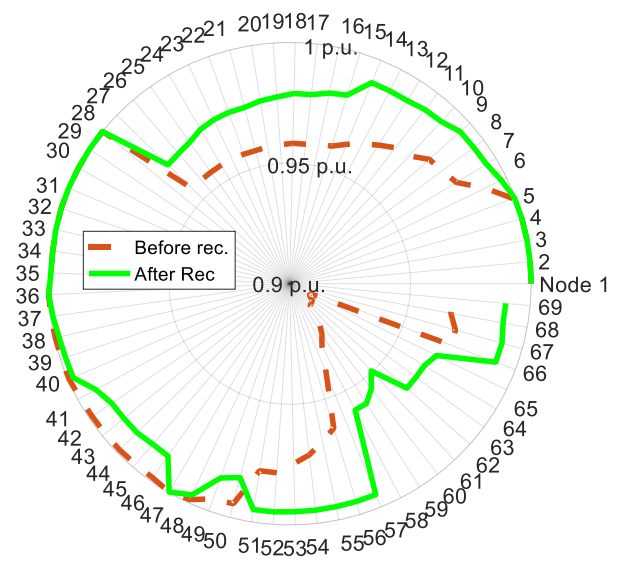

Figure 1. The voltage of nodes in the 69node test system after reconfiguration

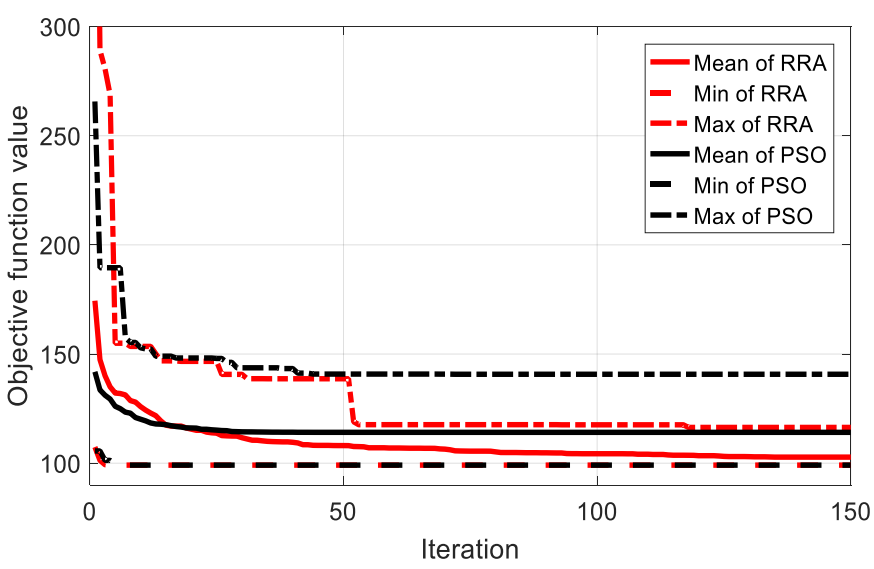

Figure 2. The convergence curves of RRA and PSO for 69-node test system

\section{CONCLUSION}

The paper demonstrates the NR method based on RRA to optimize the network configuration. The objective function of the NR problem is minimizing of power loss. The effectiveness of the RRA is evaluated on the 16 and 69-node EDS system. The obtained results are compared with PSO and other methods in literature. The obtained results in 50 runs have shown that RRA is outperformed PSO in terms of quality of the obtained optimal solution and the successful rates and the executed times. The success rate of 
RRA on 16-node and 69-node systems are respectively up to $82 \%$ and $58 \%$ while those of PSO is only $24 \%$ for both system. The simulated results are also shown that RRA is better than some others in literature. In addition, successful applying of RRA for the NR problem to decrease power loss in the 16 and 69-node system has shown that RRA is the potential method for the NR problem of reducing power loss and other objective functions.

\section{REFERENCES}

[1] A. Merlin and H. Back, "Search for a minimal loss operating spanning tree configuration in an urban power distribution system," Proceeding in 5th power system computation conf (PSCC), Cambridge, UK, vol. 1, pp. $1-18,1975$.

[2] S. Civanlar, J. J. Grainger, H. Yin, and S. S. H. Lee, "Distribution feeder reconfiguration for loss reduction," IEEE Transactions on Power Delivery, vol. 3, no. 3, pp. 1217-1223, 1988.

[3] J. Z. Zhu, "Optimal reconfiguration of electrical distribution network using the refined genetic algorithm," Electric Power Systems Research, vol. 62, no. 1, pp. 37-42, 2002, doi: 10.1016/S0378-7796(02)00041-X.

[4] R. T. Ganesh Vulasala, Sivanagaraju Sirigiri, "Feeder Reconfiguration for Loss Reduction in Unbalanced Distribution System Using Genetic Algorithm," International Journal of Electrical and Electronics Engineering, vol. 3, no. 12, pp. 754-762, 2009.

[5] P. Subburaj, K. Ramar, L. Ganesan, and P. Venkatesh, "Distribution System Reconfiguration for Loss Reduction using Genetic Algorithm,” Journal of Electrical Systems, vol. 2, no. 4, pp. 198-207, 2006.

[6] K. K. Kumar, N. Venkata, and S. Kamakshaiah, "FDR particle swarm algorithm for network reconfiguration of distribution systems," Journal of Theoretical and Applied Information Technology, vol. 36, no. 2, pp. 174-181, 2012.

[7] T. M. Khalil and A. V Gorpinich, "Reconfiguration for Loss Reduction of Distribution Systems Using Selective Particle Swarm Optimization," International Journal of Multidisciplinary Sciences and Engineering, vol. 3, no. 6, pp. 16-21, 2012.

[8] A. Y. Abdelaziz, S. F. Mekhamer, F. M. Mohammed, and M. a L. Badr, "A Modified Particle Swarm Technique for Distribution Systems Reconfiguration," The online journal on electronics and electrical engineering (OJEEE), vol. 1, no. 1, pp. 121-129, 2009.

[9] A. Mohamed Imran and M. Kowsalya, "A new power system reconfiguration scheme for power loss minimization and voltage profile enhancement using Fireworks Algorithm," International Journal of Electrical Power and Energy Systems, vol. 62, pp. 312-322, 2014, doi: 10.1016/j.ijepes.2014.04.034.

[10] A. Mohamed Imran, M. Kowsalya, and D. P. Kothari, "A novel integration technique for optimal network reconfiguration and distributed generation placement in power distribution networks," International Journal of Electrical Power and Energy Systems, vol. 63, pp. 461-472, 2014, doi: 10.1016/j.jepes.2014.06.011.

[11] T. T. Nguyen and A. V. Truong, "Distribution network reconfiguration for power loss minimization and voltage profile improvement using cuckoo search algorithm," International Journal of Electrical Power and Energy Systems, vol. 68, pp. 233-242, 2015, doi: 10.1016/j.ijepes.2014.12.075.

[12] T. T. Nguyen and T. T. Nguyen, "An improved cuckoo search algorithm for the problem of electric distribution network reconfiguration," Applied Soft Computing, vol. 84, p. 105720, 2019, doi: 10.1016/j.asoc.2019.105720.

[13] T. T. Nguyen, A. V. Truong, and T. A. Phung, "A novel method based on adaptive cuckoo search for optimal network reconfiguration and distributed generation allocation in distribution network," International Journal of Electrical Power and Energy Systems, vol. 78, pp. 801-815, 2016, doi: 10.1016/j.ijepes.2015.12.030.

[14] H. F. Kadom, A. N. Hussain, and W. K. S. Al-Jubori, "Dual technique of reconfiguration and capacitor placement for distribution system," International Journal of Electrical and Computer Engineering, vol. 10, no. 1, pp. 80-90, 2020, doi: 10.11591/ijece.v10i1.pp80-90.

[15] A. V. S. Reddy, M. D. Reddy, and M. S. K. Reddy, "Network reconfiguration of distribution system for loss reduction using GWO algorithm," International Journal of Electrical and Computer Engineering, vol. 7, no. 6, pp. 3226-3234, 2017, doi: 10.11591/ijece.v7i6.pp3226-3234.

[16] H. Hamour, S. Kamel, L. Nasrat, and J. Yu, "Distribution Network Reconfiguration Using Augmented Grey Wolf Optimization Algorithm for Power Loss Minimization," Proceedings of 2019 International Conference on Innovative Trends in Computer Engineering, ITCE 2019, pp. 450-454, 2019, doi: 10.1109/ITCE.2019.8646595.

[17] F. Merrikh-Bayat, "The runner-root algorithm: A metaheuristic for solving unimodal and multimodal optimization problems inspired by runners and roots of plants in nature," Applied Soft Computing, vol. 33, pp. 292-303, 2015, doi: 10.1016/j.asoc.2015.04.048.

[18] T. T. Nguyen, T. T. Nguyen, A. V. Truong, Q. T. Nguyen, and T. A. Phung, "Multi-objective electric distribution network reconfiguration solution using runner-root algorithm," Applied Soft Computing, vol. 52, pp. 93-108, 2017, doi: 10.1016/j.asoc.2016.12.018.

[19] A. V. Truong, T. N. Ton, T. T. Nguyen, and T. L. Duong, "Two states for optimal position and capacity of distributed generators considering network reconfiguration for power loss minimization based on runner root algorithm," Energies, vol. 12, no. 1, p. 106, pp. 1-16, 2019, doi: 10.3390/en12010106.

[20] A. Y. Abdelaziz, F. M. Mohammed, S. F. Mekhamer, and M. A. L. Badr, "Distribution Systems Reconfiguration using a modified particle swarm optimization algorithm," Electric Power Systems Research, vol. 79, pp. 1521-1530, 2009, doi: 10.1016/j.epsr.2009.05.004. 
[21] A. Y. Abdelaziz, F. M. Mohamed, S. F. Mekhamer, and M. A. L. Badr, "Distribution system reconfiguration using a modified Tabu Search algorithm," Electric Power Systems Research, vol. 80, no. 8, pp. 943-953, 2010, doi: 10.1016/j.epsr.2010.01.001.

[22] I. J. Ramirez-Rosado and J. L. Bernal-Agustin, "Genetic algorithms applied to the design of large power distribution systems," IEEE Transactions on Power Systems, vol. 13, no. 2, pp. 696-703, 1998, doi: $10.1109 / 59.667402$.

[23] A. Fathy, M. El-Arini, and O. El-Baksawy, "An efficient methodology for optimal reconfiguration of electric distribution network considering reliability indices via binary particle swarm gravity search algorithm," Neural Computing and Applications, vol. 30, pp. 2843-2858, 2017, doi: 10.1007/s00521-017-2877-z.

[24] H.-D. Chiang and R. Jean-Jumeau, "Optimal network reconfigurations in distribution systems: Part 2: Solution algorithms and numerical results," IEEE Transactions on Power Delivery, vol. 5, no. 3, pp. 1568-1574, 1990, doi: $10.1109 / 61.58002$

[25] A. Onlam, D. Yodphet, R. Chatthaworn, C. Surawanitkun, A. Siritaratiwat, and P. Khunkitti, "Power Loss Minimization and Voltage Stability Improvement in Electrical Distribution System via Network Reconfiguration and Distributed Generation Placement Using Novel Adaptive Shuffled Frogs Leaping Algorithm," Energies, vol. 12, no. 3, pp. 1-12, 2019, doi: 10.3390/en12030553.

[26] R. S. Rao, K. Ravindra, K. Satish, and S. V. L. Narasimham, "Power Loss Minimization in Distribution System Using Network Reconfiguration in the Presence of Distributed Generation," IEEE Transactions on Power Systems, vol. 28, no. 1, pp. 317-325, 2013, doi: 10.1109/TPWRS.2012.2197227. 